\title{
TNF rises to the occasion
}

A new report in the The Journal of Clinical Investigation shows that, in mice, the pro-inflammatory cytokine tumour-necrosis factor (TNF) and its receptors, TNF receptor 1 (TNFR1) and TNFR2, are not essential during a strong inflammatory response, but are crucial in the regulation of immune responses that occur under conditions of limited immunostimulatory capacity, such as tumour surveillance.

TNF-antagonist therapy is widely used to treat inflammatory and autoimmune diseases, such as rheumatoid arthritis, in which dysregulated or excessive TNF production has been implicated. However, there is evidence to suggest that TNF-specific therapy is linked to an increased incidence of malignancies in patients with rheumatoid arthritis. Understanding the consequences of TNF blockade, therefore, is important, but is complicated by contradictory reports on the role of TNF in the regulation of T-cell responses.

To address this issue, Calzascia et al. investigated whether the involvement and relevance of TNF in immunity is associated with the immunostimulatory properties of the antigen. They used the lymphocytic choriomeningitis virus (LCMV) glycoprotein as a model antigen in LCMV infection (which induces a strong inflammatory response) or in a tumour model (in which a glycoprotein-expressing spontaneous tumour acts as a stimulus associated with limited immunostimulatory capacity).

TNF-deficient mice did not show impaired antiviral immune responses, which are associated with a strong inflammatory response, but TNF was crucial for immune responses to tumour-associated antigens. TNF-TNFR1-mediated signals in antigen-presenting cells

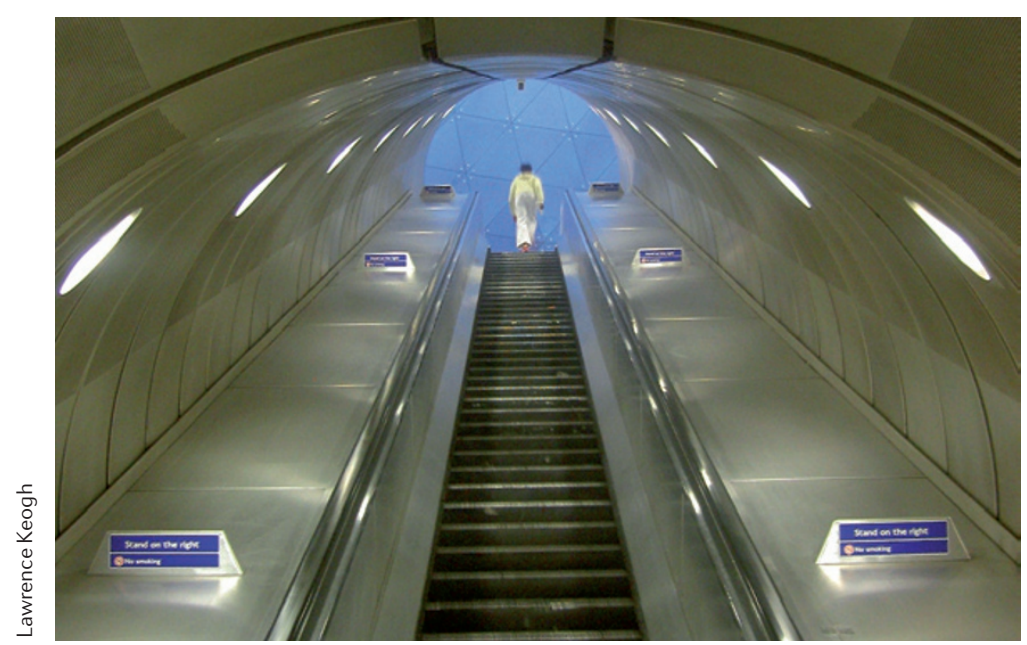

and TNF-TNFR2-mediated signals in T cells were necessary for effective priming, proliferation and recruitment of tumour-specific T cells. In TNF-deficient mice, T-cell priming and expansion in the tumour-draining lymph nodes were impaired, and antitumour immunity was abrogated.

Could some of the immunostimulatory parameters that are provided during LCMV infection (such as Toll-like receptor (TLR) triggering, $\mathrm{CD}_{4}^{+} \mathrm{T}$-cell help and systemic cytokine secretion), which are lacking in tumour-bearing animals, cooperate to render TNF dispensable? The exogenous addition of a CD40 agonist, alone or in combination with TLR2 stimulation, was shown to rescue the defective proliferation of TNF-deficient T cells in TNF-deficient, tumour-bearing mice, indicating that in their absence, TNF is crucial for activating tumourreactive T cells in this model.

This paper suggests that TNF signalling may only be essential in the response to conditions such as the development of spontaneous malignancies, in which there is limited immunostimulatory capacity. These results could have important implications in terms of tumour progression or development for patients with cancer or autoimmune disease who are treated with TNF antagonists.

Sharon Ahmad

ORIGINAL RESEARCH PAPER Calzascia, T. et al. TNF- $\alpha$ is critical for antitumor but not antiviral T cell immunity in mice. J. Clin. Invest. 8 November 2007 (doi:10.1172/JCl32567) 